\title{
World Mental Health Day 2020: Promoting Global Mental Health During COVID-19
}

\author{
Michael R. Phillips ${ }^{1,2, \#}$
}

Observed for the first time on October 10, 1992, World Mental Health Day was initiated by the World Federation for Mental Health and is currently coordinated by the World Health Organization (WHO), the World Federation for Mental Health, and the United for Global Mental Health organization. These annual events promote awareness of the importance of mental health and mobilize support for providing high-quality, community-based care to all persons who need mental health services; this year, most events will be conducted online due to the coronavirus disease 2019 (COVID-19) pandemic $(1-3)$.

\section{MENTAL HEALTH FOR ALL: GREATER INVESTMENT — GREATER ACCESS}

The theme of this year's World Mental Health Day is "Mental Health for All: Greater Investment, Greater Access". This theme highlights the chronic lack of resources for mental health and the inequitable distribution of the limited resources that are available. Despite increased awareness of the importance of mental health and psychological wellbeing to economic and social development by national governments and international agencies over the last two decades, there has not been a corresponding increase in investment in mental health. In its news release about World Mental Health Day, the WHO (4) reports that 1 billion people are currently living with mental disorders, 3 million individuals die each year of alcohol use disorder, and 1 person dies every 40 seconds by suicide. In spite of this huge toll on global productivity, $75 \%$ of people with mental disorders in low- and middle-income countries (LMICs) receive no treatment whatsoever for their condition.

Based on data from the Global Burden of Disease Study (5) in 2017, mental disorders, substance-use disorders, and self-harm accounted for $8.0 \%$ of the total global burden of disease for all health conditions and injuries, but WHO's 2017 Mental Health Atlas
(6) reports that less than $2 \%$ of government health budgets are spent on mental health and that the vast majority of these funds go to specialized mental health hospitals. Moreover, in $27 \%$ of the countries that provided data to the $\mathrm{WHO}$, mental health is not covered in national health insurance plans. This mismatch between the huge burden of mental disorders, the limited government support for mental health services, and the focus of the limited financial resources available for mental health on psychiatric hospitals (which are primarily situated in urban centers) means that in many countries individuals with mental disorders, particularly those living in rural communities of LMICs, have no access to needed mental health care.

Investment in the equitable distribution of highquality services for persons affected by mental disorders is not only an ethical imperative; it is also an economic imperative. In 2018, the Lancet Commission of Global Mental Health ( 7 ) estimated that the ongoing increase in mental health and substance use disorders around the world could cost the global economy US $\$ 16$ trillion between 2010 and 2030. However, the Commission found that rapid expansion in investment in mental health could significantly decrease the economic burden caused by mental disorders. For example, the WHO estimates that for every US\$1 invested in the treatment of common mental disorders, such as depression and anxiety, there is US $\$ 5$ return in improved health and productivity.

\section{HOW IS CHINA DOING?}

China has invested substantial resources in several major mental health initiatives over the past 15 years to improve the accessibility and quality of mental health services. The Ministry of Health $\mathrm{MOH}$, then known as National Health and Family Planning Commission, now known as National Health Commision) and Ministry of Finance of China launched the National Continuing Management and Intervention Program for 
Psychoses in 2004, which monitors the treatment of community-dwelling individuals with severe mental illnesses and provides free medication to those unable to pay for these services in 60 demonstration districts/counties. With the gradual expansion of the Program coverage, the $\mathrm{MOH}$ built the National Information System for Psychoses in 2011 to record the patients' follow-up and treatment information. As of 2017 this system was monitoring 5.4 million individuals with psychosis from all provincial-level administrative divisions (PLADs) around the country, making it the largest community-based mental health service network in the world (8). In 2012, China passed its first national mental health law, which provided a forward-looking roadmap for the development of mental health services with a clear emphasis on expanding community-based services (9). From 2013-2015 a national mental health epidemiological study involving representative samples from all 31 PLADs was conducted (10), and in 2015, as part of the $13^{\text {th }}$ National 5-Year Plan, the 2015-2020 National Mental Health Work Plan (11) provided specific numeric targets for achieving the aspirational goals outlined in the 2012 mental health law.

Implementing these programs and policies has not been easy. Despite rapid improvement in the overall national access and quality of health services over the last 20 years, China has simultaneously seen an increasing gap in the access and quality of health services - including mental health services - between the rich eastern PLADs and the more rural western PLADs (12). As has occurred in many countries, the attempt to shift most mental health services from specialized psychiatry hospitals in urban centers to general hospitals and rural health clinics has only been partially successful; and the coordination of community-based services with hospital-based services still needs to be strengthened, especially in rural areas. The attention to the prevention of mental illnesses is just beginning, and specialized mental health services for children and the elderly are only available in large cities. The vast majority of mental health resources are focused on the management of psychotic disorders, while relatively few resources are used to actively identify and treat common mental disorders such as depression and anxiety that account for a much larger proportion of the national socioeconomic burden of mental illnesses. Mental health manpower is largely limited to psychiatrists and psychiatric nurses; meeting the increasing mental health needs of the population will require expanding the responsibilities of all general physicians to include the recognition and management of common mental disorders and greatly increasing the numbers of clinical psychologists and psychiatric social workers. The success or failure of provincial-level and national mental health programs and policies must be regularly evaluated by independent groups of external experts in addition to routine internal evaluations by the institutions that are responsible for the programs, and the programs and policies need to be regularly revised and updated based on their findings.

\section{COVID-19 AND MENTAL HEALTH}

The COVID-19 pandemic of 2020 and the social and economic effects of managing the epidemic are likely to significantly affect community mental health (13). These effects will range from short-term fear and anxiety about potential infection during times of high prevalence, long-term depressive and cognitive effects in persons with chronic post-infection health conditions, pathological grieving for deceased family members who could not be visited at the time of death, the psychological effects of large-scale social and economic disruption, and increased severity of preexisting mental disorders in individuals who cannot access their usual social support or health care networks due to lockdowns or social distancing. The prevalence, severity, demographic distribution, and social and economic effects of COVID-19 vary widely by country, so it is likely that the mental health effects will also vary widely by location and by population cohort within each community. Given that the pandemic is still ongoing, research about its effects on mental health must be considered preliminary (14). For example, a review of the accumulating reports about the effect of COVID-19 on suicidal behavior (15) finds that the quality of such studies is poor; to date, there is no evidence of changes in suicide rates. Monitoring the long-term effects of COVID-19 (and other global or national crises) on mental health will require undertaking annual, high-quality epidemiological surveys of mental health disorders, substance use disorders, and suicidal behaviors in large, nationally representative samples of community residents.

At a time when the need to support the mental health of the community is the greatest due to COVID-19, the resources available to do so in most countries have decreased because the financial needs of other community services (e.g., screening for and 
treating COVID-19 infections, providing unemployment and welfare support, making schools COVID-19-safe) have increased dramatically while available government funds have been reduced due to decreased tax revenues. In this constrained economic environment, it will be difficult to convince governments and non-governmental organizations (NGOs) to earmark their limited funds and other resources for the sole purpose of increasing the access and quality of mental health services. It should, however, be feasible to include mental health issues as an essential part of governments' and NGOs' overall COVID-19 management and recovery plans, so mental health advocates will need to fight to ensure that this happens. Ideally, these challenging times could stimulate the development and promulgation of less-expensive, community-based services — such as the use of telepsychiatry or 'mental health extenders' for patients in under-served rural communities — but even if feasible, such innovations are unlikely to substantially change the dire lack of needed mental health services. Once COVID-19 vaccines are widely available, it may be possible to regain lost ground, but long-term financial damages in some LMICs will likely continue to limit the development of mental health services.

In summary, over a decade ago, global mental health advocates promoted the concept of "no health without mental health" (16). This increased awareness of the magnitude of the global mental health problem but had little effect on changing the situation. More recently, the effort to promote global mental health via World Mental Health Day and other initiatives aims to reframe mental health as an integral part of the overall social and economic development using the motto: "No sustainable development without mental health" (7). However, the COVID-19 pandemic is substantially altering the social environment and economic development of many countries, and at this point in time, no one can be certain what the postpandemic, 'new normal' will look like. Thus, the longterm effects of COVID-19 on global mental health also remain uncertain. Ongoing monitoring of the prevalence, severity, and duration of mental health conditions and of the available services for preventing and treating these conditions will be needed to determine whether or not the current strategy of global mental health advocates of linking mental health with development will result in improved global mental health.

In 2019, Dr Tedros Adhanom Ghebreyesus,
Director-General of the WHO clarified the central role of mental health to global health as follows: "The world is accepting the concept of universal health coverage(UHC). Mental health must be an integral part of UHC. Nobody should be denied access to mental health care because she or he is poor or lives in a remote place" (17).

Conflicts of interest: The author reports no conflicts of interest related to this manuscript.

doi: $10.46234 / \mathrm{ccdcw} 2020.220$

\# Corresponding author: Michael R. Phillips, mphillipschina@ outlook.com.

${ }^{1}$ Shanghai Mental Health Center, Shanghai Jiaotong University School
of Medicine, Shanghai, China; ${ }^{2}$ Departments of Psychiatry and
Epidemiology, Columbia University, New York, NY, USA.

Submitted: October 05, 2020; Accepted: October 09, 2020

\section{REFERENCES}

1. World Mental Health Day 2020. WHO. https://www.afro.who.int/ regional-director/speeches-messages/world-mental-health-day-2020. [2020-10-05].

2. Are you ready to move for mental health? United for Global Mental Health. https://unitedgmh.org/are-you-ready-move-mental-health. [2020-10-05].

3. World Mental Health Day 2020: mental health for all: greater investment-greater access. World Federation for Mental Health. https://wfmh.global/wp-content/uploads/WMHD2020_v16_resized. pdf. [2020-10-05]

4. World Mental Health Day: an opportunity to kick-start a massive scaleup in investment in mental health. WHO News Release, 27 August 2020. https://www.who.int/news-room/detail/27-08-2020world-mental-health-day-an-opportunity-to-kick-start-a-massive-scaleup-in-investment-in-mental-health. [2020-10-05].

5. Institute for health metrics and evaluation. https://vizhub.healthdata. org/gbd-compare. [2020-10-05].

6. WHO. Mental health ATLAS 2017. WHO; 2018. https://www.who. int/mental_health/evidence/atlas/mental_health_atlas_2017/en/. [202010-05].

7. Patel V, Saxena S, Lund C, Thornicroft G, Baingana F, Bolton P, et al. The Lancet Commission on global mental health and sustainable development. Lancet 2018;392(10157):1553 - 98. http://dx.doi.org/10. 1016/S0140-6736(18)31612-X.

8. Wu XM, Ma N, Wang X, Zhang WF, Guan LL, Ma H, et al. Management and services for psychosis in People's Republic of China in 2017. Chin J Psychiatry 2019;52(1):82 - 8. http://dx.doi.org/10. 3760/cma.j.issn.1006-7884.2019.01.008. (In Chinese).

9. Chen HH, Phillips MR, Cheng H, Chen QQ, Chen XD, Fralick D, et al. Mental health law of the People's Republic of China (English translation with annotations). Shanghai Arch Psychiatry 2012;24 (6):305 - 21. http://dx.doi.org/10.3969/j.issn.1002-0829.2012.06.001.

10. Huang YQ, Wang Y, Wang H, Liu ZR, Yu X, Yan J, et al. Prevalence of mental disorders in China: a cross-sectional epidemiological study. Lancet Psychiatry 2019;6(3):211 - 4. http://dx.doi.org/10.1016/S22150366(18)30511-X

11. Xiong W, Phillips MR. Translated and annotated version of the 20152020 National Mental Health Work Plan of the People's Republic of China. Shanghai Arch Psychiatry 2016;28(1):4 - 17. http://dx.doi.org/ 10.11919/j.issn.1002-0829.216012.

12. GBD 2016 Healthcare Access and Quality Collaborators. Measuring performance on the healthcare access and quality index for 195 
countries and territories and selected subnational locations: a systematic analysis from the Global Burden of Disease Study 2016. Lancet 2018;391(10136):2236 - 2271. http://dx.doi.org/10.1016/S0140-6736 (18)30994-2.

13. United Nations. Policy brief: COVID-19 and the need for action on mental health. 2020. https://www.un.org/sites/un2.un.org/files/un_ policy_brief-covid_and_mental_health_final.pdf. [2020-10-05].

14. Moreno C, Wykes T, Galderisi S, Nordentof M, Crossley N, Jones N, et al. How mental health care should change as a consequence of the COVID-19 pandemic. Lancet Psychiatry 2020;7(9):813- 24. http://dx.doi.org/10.1016/S2215-0366(20)30307-2.

15. John A, Okolie C, Eyles E, Webb RT, Schmidt L, McGuiness LA, et al.
The impact of the COVID-19 pandemic on self-harm and suicidal behaviour: a living systematic review [version 1; peer review: 1 approved]. F1000Research 2020;9:1097. http://dx.doi.org/10.12688/ f1000research.25522.1.

16. Prince M, Patel V, Saxena S, Maj M, Maselko J, Phillips MR, et al. No health without mental health. Lancet 2007;370(9590):859-77. http://dx.doi.org/10.1016/S0140-6736(07)61238-0.

17. WHO. Special initiative for mental health (2019-2023): universal health coverage for mental health. Geneva: WHO; 2019. https://www. who.int/mental_health/evidence/special_initiative_2019_2023/en. [2020-10-05]

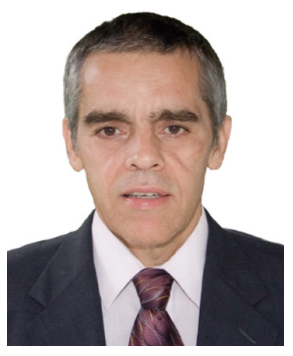

Michael R. Phillips CM, MD, MA, MPH Professor of Psychiatry, Shanghai Jiao Tong University School of Medicine Professor of Clinical Psychiatry and Clinical Epidemiology, Columbia University Director, Suicide Research and Prevention Center, Shanghai Mental Health Center Executive Director, WHO Collaborating Center for Research and Training in Suicide Prevention, Beijing Huilongguan Hospital Co-Chairman Lancet Commission on Mental Health in China. 